Please do not remove this page

RMIT

UNIVERSITY

\title{
Optimal design of magnitude responses of rational infinite impulse response filters
}

Ho, Charlotte; Ling, Bingo Wing-Kuen; Liu, Yanqun; Tam, Peter; Teo, Kok Lay

https://researchrepository.rmit.edu.au/esploro/outputs/9921859773601341/filesAndLinks?institution=61 RMIT_INST\&index=null

Ho, C., Ling, B. W.-K., Liu, Y., Tam, P., \& Teo, K. L. (2006). Optimal design of magnitude responses of rational infinite impulse response filters. IEEE Transactions on Signal Processing, 54(10), 4039-4046. https://doi.org/10.1109/TSP.2006.880317

Published Version: https://doi.org/10.1109/TSP.2006.880317

Repository homepage: https://researchrepository.rmit.edu.au

(c) 2006 IEEE. Personal use of this material is permitted. However, permission to reprint/republish this material for advertising or promotional purposes or for creating new collective works for resale or redistribution to servers or lists, or to reuse any copyrighted component of this work in other works must be obtained from the IEEE.

Downloaded On 2023/04/26 19:54:43 +1000 
their constructive comments and suggestions that have significantly improved the manuscript.

\section{REFERENCES}

[1] S. Haykin, Adaptive Filter Theory, 3rd ed. Englewood Cliffs, NJ: Prentice-Hall, 1996.

[2] G. H. Golub and C. F. van Loan, Matrix Computations. Baltimore, MD: The Johns Hopkins Univ. Press, 1983.

[3] R. Vaccaro, "On adaptive implementations of Pisarenko's harmonic retrieval method," in Proc. IEEE Int. Conf. Acoustics, Speech, Signal Processing (ICASSP), San Diego, CA, Mar. 1984, vol. 9, pp. 224-227.

[4] H. Chen, T. K. Sarkar, S. A. Dianat, and J. D. Brule, "Adaptive spectral estimation by the conjugate gradient method," IEEE Trans. Acoust., Speech, Signal Process., vol. ASSP-34, no. 2, pp. 272-284, Apr. 1986.

[5] V. U. Reddy, B. Egardt, and T. Kailath, "Least squares-type algorithm for adaptive implementations of Pisarenko's harmonic retrieval method," IEEE Trans. Acoust., Speech, Signal Process., vol. ASSP-34, no. 3, pp. 399-405, Jun. 1982.

[6] C. E. Davila, "An efficient recursive total least squares algorithm for FIR adaptive filtering," IEEE Trans. Signal Process., vol. 42, no. 2, pp. 268-280, Feb. 1994.

[7] L. Ljung, M. Morf, and D. Falconer, "Fast calculation of gain matrices for recursive estimation schemes," Int. J. Control, vol. 27, no. 1, pp. $1-19,1978$.

[8] S. Ljung and L. Ljung, "Error propagation properties of recursive least-squares adaptation algorithms," Automatica, vol. 21, no. 2, pp. 157-167, Mar. 1985.

[9] J. L. Botto and G. V. Moustakides, "Stabilization of fast Kalman algorithms," IEEE Trans. Acoust., Speech Signal Process., vol. ASSP-37, no. 9, pp. 1342-1348, Sep. 1989.

[10] D. T. Slock and T. Kailath, "Numerically stable fast transversal filters for recursive least squares adaptive filtering," IEEE Trans. Signal Process., vol. 39, no. 1, pp. 92-114, Jan. 1991.

[11] C. E. Davila, "Line search algorithms for adaptive filtering," IEEE Trans. Signal Process., vol. 41, no. 7, pp. 2490-2494, Jul. 1994.

[12] D.-Z. Feng, X.-D. Zhang, D.-X. Chang, and W. X. Zheng, "A fast recursive total least squares algorithm for adaptive FIR filtering," IEEE Trans. Signal Process., vol. 52, no. 10, pp. 2729-2737, Oct. 2004.

[13] B. E. Dunne and G. A. Williamson, "QR-based TLS and mixed LS-TLS algorithms with applications to adaptive IIR filtering," IEEE Trans. Signal Process., vol. 51, no. 2, pp. 386-394, Feb. 2003.

[14] B. E. Dunne and G. A. Williamson, "Analysis of gradient algorithms for TLS-based adaptive IIR filters," IEEE Trans. Signal Process., vol. 52, no. 12, pp. 3345-3356, Dec. 2004.

[15] A. Yeredor, "The extended least squares criterion: Minimization algorithms and applications," IEEE Trans. Signal Process., vol. 49, no. 1, pp. 74-86, Jan. 2001.

[16] Y. N. Rao, D. Erdogmus, and J. C. Principe, "Error whitening criterion for adaptive filtering: Theory and algorithms," IEEE Trans. Signal Process., vol. 53, no. 3, pp. 1057-1069, Mar. 2005.

[17] J. J. Hopfield, "Neural networks and physical systems with emergent collective computational abilities," Proc. Nat. Acad. Sci. USA, vol. 79, pp. 2554-2558, 1982.

[18] K. H. Huebner and E. A. Thornton, The Finite Element Method for Engineers. New York: Wiley, 1982.

\section{Optimal Design of Magnitude Responses of Rational Infinite Impulse Response Filters}

Charlotte Yuk-Fan Ho, Bingo Wing-Kuen Ling, Yan-Qun Liu, Peter Kwong-Shun Tam, and Kok-Lay Teo

\begin{abstract}
This correspondence considers a design of magnitude responses of optimal rational infinite impulse response (IIR) filters. The design problem is formulated as an optimization problem in which a total weighted absolute error in the passband and stopband of the filters (the error function reflects a ripple square magnitude) is minimized subject to the specification on this weighted absolute error function defined in the corresponding passband and stopband, as well as the stability condition. Since the cost function is nonsmooth and nonconvex, while the constraints are continuous, this kind of optimization problem is a nonsmooth nonconvex continuous functional constrained problem. To address this issue, our previous proposed constraint transcription method is applied to transform the continuous functional constraints to equality constraints. Then the nonsmooth problem is approximated by a sequence of smooth problems and solved via a hybrid global optimization method. The solutions obtained from these smooth problems converge to the global optimal solution of the original optimization problem. Hence, small transition bandwidth filters can be obtained.
\end{abstract}

Index Terms-Constraint transcription method, hybrid global optimization method, rational infinite-impulse-response (IIR) filters.

\section{INTRODUCTION}

Although it is more difficult for rational infinite-impulse-response (IIR) filters to have linear phase frequency responses when compared with that for finite-impulse-response (FIR) filters, costs for implementing the rational IIR filters are usually lower than that for the FIR filters at given passband and stopband specifications. Hence, rational IIR filters are preferred in many industrial and engineering applications in which phase responses are not very important [1]-[3]. In particular, in a sigma-delta modulator, it consists of a discrete-time filter. Since a sigma-delta modulator is operated in an oversampling manner, a narrowband filter is required. As a result, a rational IIR filter is preferred because the cost for employing an FIR filter is too high.

Manuscript received April 19, 2005; revised November 25, 2005. The associate editor coordinating the review of this correspondence and approving it for publication was Prof. Mariane R. Petraglia. The work obtained in this correspondence was supported by a research grant (project number G-YD26) from The Hong Kong Polytechnic University, the Centre for Multimedia Signal Processing, The Hong Kong Polytechnic University, the CRGC Grant (project number PolyU 5105101E) from the Research Grants Council of Hong Kong, as well as a research grant from Australian Research Council.

C. Y.-F. Ho is with the Department of Electronic Engineering, Queen Mary, University of London, London E1 4NS, U.K. (e-mail: charlotte.ho@elec.qmul. ac.uk).

B. W.-K. Ling is with the Department of Electronic Engineering, Division of Engineering, King's College London, London, WC2R 2LS, U.K. (e-mail wing-kuen.ling@kcl.ac.uk).

Y.-Q. Liu is with the Department of Mathematics and Statistics, Royal Melbourne Institute of Technology, Melbourne VIC 3001, Australia. (e-mail: yanqun.liu@rmit.edu.au).

P. K.-S. Tam is with the Department of Electronic and Information Engineering, The Hong Kong Polytechnic University, Kowloon, Hong Kong, China (e-mail: enptam@polyu.edu.hk).

K.-L. Teo is with the Department of Mathematics and Statistics, Curtin University of Technology, Perth, 00301J, Australia (e-mail: K.L.Teo@curtin.edu. au).

Color versions of Figs. 1-7 are available online at http://ieeexplore.ieee.org. Digital Object Identifier 10.1109/TSP.2006.880317 
Due to the quantization process, the phase information is seriously corrupted by the quantizer. Hence, the phase information cannot be exploited, and it is not very important for the design of a sigma-delta modulator.

One of the most common methods for designing rational IIR filters is via eigenfilter approaches [4]-[8], in which optimal solutions can be found by computing the eigenvalues of the error matrices. Another method is via a WISE approach [19]. An optimal solution can be found by computing a gradient of the corresponding cost function. A model-matching approach [17] was also proposed. This method is to model rational IIR filters as FIR filters and then minimized the difference between a norm of these two classes of filters. However, since all these methods [4]-[8], [17], [19] are based on formulating their design problems as unconstrained optimization problems, the stability, as well as the size of the ripple magnitudes in passbands and stopbands of the filters, are not guaranteed. Moreover, they required phase information for the desired filter responses. In some applications, such as the applications in sigma-delta modulators [1], phase responses are not very important. Imposing extra phase information on desired filter responses may cause degradation on filter performances.

In order to tackle parts of these issues, rational IIR filter design problems are formulated as constrained optimization problems subject to various constraints. These optimization problems are solved via the Gauss-Newton method [18]. However, this method replied on smooth cost functions and is easy to trap at local minima because these optimization problems are not convex. In order to avoid computing the gradients of cost functions, these design problems are formulated as constrained iterative design problems [9]-[16]. Filter coefficients are designed based on initialized denominator coefficients and the iteration of the design process until the denominator coefficients converged. Since these approaches required an initialization of denominator coefficients, the global optimal solutions, as well as the convergence of the iterative process, are not guaranteed.

There were some other methods proposed for designing rational IIR filters, such as via halfband filters [20]. However, this approach is not applied if filters are not halfband ones. Another method based on controlling frequency response of filters continuously was proposed [21]. As it is a kind of adaptive filter design techniques, the filters are time varying.

If only the magnitude response of rational IIR filters is designed, then we can formulate the design problems as optimization problems. The cost of the corresponding optimization problems can be defined as the total weighted absolute error in the passbands and stopbands of the filters, in which the error function reflects the ripple square magnitudes, subject to constraints based on the specification on this weighted absolute error function in the corresponding passbands and stopbands, as well as to a stability condition of the filters. However, this kind of optimization problem is difficult to solve because it involves a nonsmooth nonconvex cost and continuous functional constraints.

To solve the optimization problems with continuous functional constraints, one may sample these continuous functional constraints and convert to finite discrete constraints [14], [22]. However, it is not guaranteed that solutions obtained satisfy the original continuous functional constraints. Although the difference between the exact upper bounds of discretized constraint functions and that of the corresponding continuous functional constraint functions decrease as the number of grid points increases, the computational complexity increases. To find the global optimal solution of nonconvex problems, one may apply the bridging method [23]. However, this method is applied only for one-dimensional optimization problems.

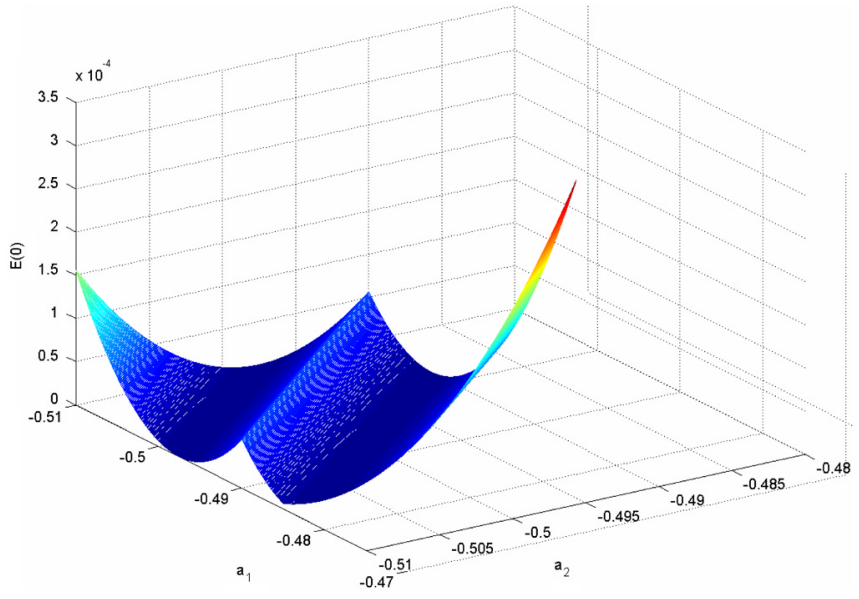

Fig. 1. Plot of $E(0)$ against different denominator coefficients. It can be seen that $E(0)$ is not differentiable with respect to the denominator coefficients.

In this correspondence, a magnitude design of rational IIR filters is formulated as a nonsmooth nonconvex optimization problem with continuous functional constraints. Our previous proposed constraint transcription method [24] is applied to transform these continuous functional constraints to equality constraints. The global optimal solution can be obtained via the hybrid global optimization method [25]. The obtained numerical experiments show that very small transition bandwidth filters can be obtained.

The outline of this correspondence is as follows. The problem formulation is presented in Section II. The numerical experiments are shown in Section III. Finally, a conclusion is summarized in Section IV.

\section{PROBLEM Formulation}

Consider a general rational IIR filter with frequency response

$$
H(\omega)=\frac{e^{-j D \omega} \sum_{m=0}^{M} b_{m} e^{-j m \omega}}{1+\sum_{n=1}^{N} a_{n} e^{-j n \omega}}
$$

where $j \equiv \sqrt{-1}, D$ relates to the delay of the filter, $M$ and $N$ are, respectively, the number of nonzero roots of the polynomials of $e^{-j \omega}$ in the numerator and denominator, $b_{m}$ for $m=0,1, \ldots, M$ and $a_{n}$ for $n=1,2, \ldots, N$ are, respectively, the filter coefficients in the numerator and denominator. Solving the optimal filter design problem is equivalent to determine the values of $a_{n}$ for $n=1,2, \ldots, N$ and $b_{m}$ for $m=0,1, \ldots, M$. It is worth noting that $D \in \Re$ is not important for the magnitude design problem, where $\Re$ denotes the set of all real numbers. Here, we only consider filters with real coefficients, which are the most usual cases in most applications [1]-[3]. Therefore, $a_{n}, b_{m}, \in \Re$ for $n=1,2, \ldots, N$ and $m=0,1, \ldots, M$. It is worth noting that both the causal and noncausal filters can be designed via the following approach. That means $M$ can be greater than, equal, or less than $N, D$ can be positive, zero or negative numbers, and not necessarily an integer.

Let the desired magnitude response of $H(\omega)$ be $\tilde{H}(\omega)$, where $\tilde{H}(\omega) \geq 0, \forall \omega \in[-\pi, \pi]$. We want to achieve

$$
\left|\frac{e^{-j D \omega} \sum_{m=0}^{M} b_{m} e^{-j m \omega}}{1+\sum_{n=1}^{N} a_{n} e^{-j n \omega}}\right|^{2} \approx(\tilde{H}(\omega))^{2}
$$


where $|\cdot|$ denotes the modulus of the corresponding complex function. There are many ways to formulate an error function. For example, we can formulate an error function as follows:

$E(\omega) \equiv\left(\left|e^{-j D \omega} \sum_{m=0}^{M} b_{m} e^{-j m \omega}\right|-\tilde{H}(\omega)\left|1+\sum_{n=1}^{N} a_{n} e^{-j n \omega}\right|\right)^{2}$.

However, this function is not differentiable with respect to the filter coefficients. For example, consider a second-order rational IIR filter with $D=0, b_{0}=2.816335701763035 \times 10^{-3}, b_{1}=$ $1.877557134508662 \times 10^{-3}$ and $b_{2}=2.816335701763063 \times 10^{-3}$. The plot of $E(\omega)$ against $\left(a_{1}, a_{2}\right)$ at $\omega=0$ is shown in Fig. 1. It can be seen from the figure that $E(0)$ is not differentiable along the line $a_{1}+a_{2}+1=0$. Besides, since this error function consists of taking the modulus operators inside the square operator, there does not exist any method for solving this kind of nonsmooth problem. Although there are some alternative methods to define the error function so that the error function is smooth, for example, $E(\omega) \equiv$ $\left(\left|e^{-j D \omega} \sum_{m=0}^{M} b_{m} e^{-j m \omega}\right|^{2}-\tilde{H}(\omega)\left|1+\sum_{n=1}^{N} a_{n} e^{-j n \omega}\right|^{2}\right)^{2}$, the error function is fourth order, and many local minima and maxima would be occurred. In order to tackle this issue, we redefine $E(\omega)$ as follows:

$E(\omega) \equiv\left|e^{-j D \omega} \sum_{m=0}^{M} b_{m} e^{-j m \omega}\right|^{2}-(\tilde{H}(\omega))^{2}\left|1+\sum_{n=1}^{N} a_{n} e^{-j n \omega}\right|^{2}$.

In this case, $E(\omega)$ is differentiable with respect to the filter coefficients. Let the filter coefficients in the numerator and denominator be, respectively,

$$
\mathbf{x}_{n} \equiv\left[b_{0}, b_{1}, \ldots, b_{M}\right]^{T}
$$

and

$$
\mathbf{x}_{d} \equiv\left[a_{1}, a_{2}, \ldots, a_{N}\right]^{T}
$$

where the superscript ${ }^{T}$ denotes the transpose. Define

$$
\boldsymbol{\eta}_{n}(\omega) \equiv\left[1, e^{-j \omega}, \ldots, e^{-j M \omega}\right]^{T}
$$

and

$$
\boldsymbol{\eta}_{d}(\omega) \equiv\left[e^{-j \omega}, e^{-j 2 \omega}, \ldots, e^{-j N \omega}\right]^{T}
$$

then

$$
E(\omega)=\left|\left(\boldsymbol{\eta}_{n}(\omega)\right)^{T} \mathbf{x}_{n}\right|^{2}-(\tilde{H}(\omega))^{2}\left|1+\left(\boldsymbol{\eta}_{d}(\omega)\right)^{T} \mathbf{x}_{d}\right|^{2} .
$$

Denote the passband and stopband of the filter be, respectively, $B_{P}$ and $B_{S}$. In order to design a rational IIR filter having good frequency selectivity, the total ripple energy in both the passband and stopband of the filter should be minimized. Hence, we define a cost function as follows:

$$
\tilde{J}\left(\mathbf{x}_{n}, \mathbf{x}_{d}\right) \equiv \int_{B_{P} \cup B_{S}} W(\omega)|E(\omega)| d \omega
$$

where $W(\omega)>0, \forall \omega \in B_{P} \cup B_{S}$ is a weighting function. This cost function can represent the total weighted absolute ripple square magnitude in the passband and stopband of the filter because $|E(\omega)|$ represents the absolute ripple square magnitude. It is worth noting that $|E(\omega)|$ is still a nonsmooth function. However, since the modulus operator is taken outside a smooth function, this kind of optimization problem can be solved via the constraint transcription method [24] and will be discussed below.

Although the cost function can be used to minimize the total weighted absolute ripple square magnitude in the passband and stopband of the filter, there may have a very serious overshoot. Hence, a specification based on the weighted absolute ripple square magnitude is defined as follows:

$$
\tilde{W}(\omega)|E(\omega)| \leq \tilde{\delta}(\omega), \quad \forall \omega \in B_{P} \cup B_{S}
$$

where $\tilde{W}(\omega)>0, \forall \omega \in B_{P} \cup B_{S}$ is a weighting function and $\tilde{\delta}(\omega)>$ $0, \forall \omega \in B_{P} \cup B_{S}$ relates to the allowable weighted absolute ripple square magnitude in both the passband and stopband of the filter. This constraint is equivalent to

$$
\tilde{W}(\omega) E(\omega) \leq \tilde{\delta}(\omega), \quad \forall \omega \in B_{P} \cup B_{S}
$$

and

$$
-\tilde{\delta}(\omega) \leq \tilde{W}(\omega) E(\omega), \quad \forall \omega \in B_{P} \cup B_{S} .
$$

In order to guarantee that the designed filter is stable, we need to satisfy the following condition:

$$
\operatorname{Re}\left(1+\left(\boldsymbol{\eta}_{d}(\omega)\right)^{T} \mathbf{x}_{d}\right)<0, \quad \forall \omega \in[-\pi, \pi]
$$

Hence, the rational IIR filter design problem can be formulated as the following optimization problem.

\section{Problem $\mathbf{P}$ :}

$$
\begin{aligned}
& \min _{\left(\mathbf{x}_{n}, \mathbf{x}_{d}\right)} \tilde{J}\left(\mathbf{x}_{n}, \mathbf{x}_{d}\right) \equiv \int_{B_{P} \cup B_{S}} W(\omega)|E(\omega)| d \omega \\
& \text { subject to } \quad \tilde{g}_{1}\left(\mathbf{x}_{n}, \mathbf{x}_{d}, \omega\right) \equiv \tilde{W}(\omega) E(\omega)-\tilde{\delta}(\omega) \leq 0, \\
& \forall \omega \in B_{P} \cup B_{S} \\
& \tilde{g}_{2}\left(\mathbf{x}_{n}, \mathbf{x}_{d}, \omega\right) \equiv-\tilde{W}(\omega) E(\omega)-\tilde{\delta}(\omega) \leq 0, \\
& \forall \omega \in B_{P} \cup B_{S} \\
& \tilde{g}_{3}\left(\mathbf{x}_{d}, \omega\right) \equiv \operatorname{Re}\left(1+\left(\boldsymbol{\eta}_{d}(\omega)\right)^{T} \mathbf{x}_{d}\right) \leq 0, \\
& \forall \omega \in[-\pi, \pi] .
\end{aligned}
$$

It is worth noting that problem $\tilde{\mathbf{P}}$ consists of a nonsmooth nonconvex cost and continuous functional constraints. This kind of optimization problem is difficult to solve. In order to tackle this issue, our proposed constraint transcription method [24] is applied to convert these continuous functional constraints to equality constraints and discussed as follows. Since

$\max \left\{\tilde{g}_{1}\left(\mathbf{x}_{n}, \mathbf{x}_{d}, \omega\right), 0\right\}$

$$
= \begin{cases}0, & \tilde{g}_{1}\left(\mathbf{x}_{n}, \mathbf{x}_{d}, \omega\right) \leq 0 \\ \text { positive value, }, & \tilde{g}_{1}\left(\mathbf{x}_{n}, \mathbf{x}_{d}, \omega\right)>0\end{cases}
$$

by defining

$$
\hat{g}_{1}\left(\mathbf{x}_{n}, \mathbf{x}_{d}\right) \equiv \int_{B_{P} \cup B_{S}}\left(\max \left\{\tilde{g}_{1}\left(\mathbf{x}_{n}, \mathbf{x}_{d}, \omega\right), 0\right\}\right)^{2} d \omega
$$


then we have

$$
\begin{aligned}
& \hat{g}_{1}\left(\mathbf{x}_{n}, \mathbf{x}_{d}\right) \\
& \quad=\left\{\begin{array}{ll}
0, & \forall \omega \in B_{P} \cup B_{S}, \tilde{g}_{1}\left(\mathbf{x}_{n}, \mathbf{x}_{d}, \omega\right) \leq 0 \\
\text { positive value, } & \exists \omega \in B_{P} \cup B_{S}, \tilde{g}_{1}\left(\mathbf{x}_{n}, \mathbf{x}_{d}, \omega\right)>0
\end{array} .\right.
\end{aligned}
$$

Hence, the satisfaction of the constraint defined by $\forall \omega \in$ $B_{P} \cup B_{S} \tilde{g}_{1}\left(\mathbf{x}_{n}, \mathbf{x}_{d}, \omega\right) \leq 0$ is equivalent to the equality constraint defined by $\hat{g}_{1}\left(\mathbf{x}_{n}, \mathbf{x}_{d}\right)=0$. Since

$$
\begin{aligned}
& \left(\max \left\{\tilde{g}_{1}\left(\mathbf{x}_{n}, \mathbf{x}_{d}, \omega\right), 0\right\}\right)^{2} \\
& \quad= \begin{cases}0, & \tilde{g}_{1}\left(\mathbf{x}_{n}, \mathbf{x}_{d}, \omega\right) \leq 0 \\
\left(\tilde{g}_{1}\left(\mathbf{x}_{n}, \mathbf{x}_{d}, \omega\right)\right)^{2}, & \tilde{g}_{1}\left(\mathbf{x}_{n}, \mathbf{x}_{d}, \omega\right)>0\end{cases} \\
& \nabla_{\left(\mathbf{x}_{n}, \mathbf{x}_{d}\right)\left(\max \left\{\tilde{g}_{1}\left(\mathbf{x}_{n}, \mathbf{x}_{d}, \omega\right), 0\right\}\right)^{2}} \\
& \quad=\left\{\begin{array}{ll}
\mathbf{0}, & \tilde{g}_{1}\left(\mathbf{x}_{n}, \mathbf{x}_{d}, \omega\right)<0 \\
2 \tilde{g}_{1}\left(\mathbf{x}_{n}, \mathbf{x}_{d}, \omega\right) \nabla_{\left(\mathbf{x}_{n}, \mathbf{x}_{d}\right)} \tilde{g}_{1}\left(\mathbf{x}_{n}, \mathbf{x}_{d}, \omega\right), & \tilde{g}_{1}\left(\mathbf{x}_{n}, \mathbf{x}_{d}, \omega\right)>0
\end{array} .\right.
\end{aligned}
$$

As $2 \tilde{g}_{1}\left(\mathbf{x}_{n}, \mathbf{x}_{d}, \omega\right) \nabla_{\left(\mathbf{x}_{n}, \mathbf{x}_{d}\right)} \tilde{g}_{1}\left(\mathbf{x}_{n}, \mathbf{x}_{d}, \omega\right)=\mathbf{0}$ when $\tilde{g}_{1}\left(\mathbf{x}_{n}, \mathbf{x}_{d}, \omega\right)=0$, so $\nabla_{\left(\mathbf{x}_{n}, \mathbf{x}_{d}\right)}\left(\max \left\{\tilde{g}_{1}\left(\mathbf{x}_{n}, \mathbf{x}_{d}, \omega\right), 0\right\}\right)^{2}$ is continuous at $\tilde{g}_{1}\left(\mathbf{x}_{n}, \mathbf{x}_{d}, \omega\right) \stackrel{0}{=}$. Moreover, since $2 \max \left\{\tilde{g}_{1}\left(\mathbf{x}_{n}, \mathbf{x}_{d}, \omega\right), 0\right\} \nabla_{\left(\mathbf{x}_{n}, \mathbf{x}_{d}\right)} \tilde{g}_{1}\left(\mathbf{x}_{n}, \mathbf{x}_{d}, \omega\right) \quad \mathbf{0}$ when $\tilde{g}_{1}\left(\mathbf{x}_{n}, \mathbf{x}_{d}, \omega\right)<0$ and $2 \max \left\{\tilde{g}_{1}\left(\mathbf{x}_{n}, \mathbf{x}_{d}, \omega\right), 0\right\}$ $\times \nabla_{\left(\mathbf{x}_{n}, \mathbf{x}_{d}\right)} \tilde{g}_{1}\left(\mathbf{x}_{n}, \mathbf{x}_{d}, \omega\right)=2 \tilde{g}_{1}\left(\mathbf{x}_{n}, \mathbf{x}_{d}, \omega\right) \nabla_{\left(\mathbf{x}_{n}, \mathbf{x}_{d}\right)} \tilde{g}_{1}\left(\mathbf{x}_{n}, \mathbf{x}_{d}, \omega\right)$ when $\tilde{g}_{1}\left(\mathbf{x}_{n}, \mathbf{x}_{d}, \omega\right)>0$, so we have

$$
\begin{aligned}
\nabla_{\left(\mathbf{x}_{n}, \mathbf{x}_{d}\right)}( & \left.\max \left\{\tilde{g}_{1}\left(\mathbf{x}_{n}, \mathbf{x}_{d}, \omega\right), 0\right\}\right)^{2} \\
& =2 \max \left\{\tilde{g}_{1}\left(\mathbf{x}_{n}, \mathbf{x}_{d}, \omega\right), 0\right\} \nabla_{\left(\mathbf{x}_{n}, \mathbf{x}_{d}\right)} \tilde{g}_{1}\left(\mathbf{x}_{n}, \mathbf{x}_{d}, \omega\right) .
\end{aligned}
$$

As a result, we have

$$
\begin{aligned}
& \nabla_{\left(\mathbf{x}_{n}, \mathbf{x}_{d}\right)} \hat{g}_{1}\left(\mathbf{x}_{n}, \mathbf{x}_{d}\right) \\
& \quad=2 \int_{B_{P} \cup B_{S}} \max \left\{\tilde{g}_{1}\left(\mathbf{x}_{n}, \mathbf{x}_{d}, \omega\right), 0\right\} \nabla_{\left(\mathbf{x}_{n}, \mathbf{x}_{d}\right)} \tilde{g}_{1}\left(\mathbf{x}_{n}, \mathbf{x}_{d}, \omega\right) d \omega .
\end{aligned}
$$

Similarly, by defining

$$
\hat{g}_{2}\left(\mathbf{x}_{n}, \mathbf{x}_{d}\right) \equiv \int_{B_{P} \cup B_{S}}\left(\max \left\{\tilde{g}_{2}\left(\mathbf{x}_{n}, \mathbf{x}_{d}, \omega\right), 0\right\}\right)^{2} d \omega
$$

and

$$
\hat{g}_{3}\left(\mathbf{x}_{d}\right) \equiv \int_{[-\pi, \pi]}\left(\max \left\{\tilde{g}_{3}\left(\mathbf{x}_{d}, \omega\right), 0\right\}\right)^{2} d \omega
$$

we have

$$
\begin{aligned}
\nabla_{\left(\mathbf{x}_{n}, \mathbf{x}_{d}\right)} \hat{g}_{2}\left(\mathbf{x}_{n}, \mathbf{x}_{d}\right) & \\
= & 2 \int_{B_{P} \cup B_{S}} \max \left\{\tilde{g}_{2}\left(\mathbf{x}_{n}, \mathbf{x}_{d}, \omega\right), 0\right\} \\
& \times \nabla_{\left(\mathbf{x}_{n}, \mathbf{x}_{d}\right)} \tilde{g}_{2}\left(\mathbf{x}_{n}, \mathbf{x}_{d}, \omega\right) d \omega
\end{aligned}
$$

and

$$
\nabla_{\mathbf{x}_{d}} \hat{g}_{3}\left(\mathbf{x}_{d}\right)=2 \int_{[-\pi, \pi]} \max \left\{\tilde{g}_{3}\left(\mathbf{x}_{d}, \omega\right), 0\right\} \nabla_{\mathbf{x}_{d}} \tilde{g}_{3}\left(\mathbf{x}_{d}, \omega\right) d \omega .
$$

As $\hat{g}_{1}\left(\mathbf{x}_{n}, \mathbf{x}_{d}\right), \hat{g}_{2}\left(\mathbf{x}_{n}, \mathbf{x}_{d}\right)$, and $\hat{g}_{3}\left(\mathbf{x}_{d}\right)$ are continuously differentiable with respect to $\left(\mathbf{x}_{n}, \mathbf{x}_{d}\right)$ and $\mathbf{x}_{d}$, respectively, the optimization problem $\tilde{\mathbf{P}}$ is equivalent to the following optimization problem, denoted as problem $\mathbf{P}$.

Problem $(\mathbf{P})$ :

$$
\begin{aligned}
\min _{\left(\mathbf{x}_{n}, \mathbf{x}_{d}\right)} \quad \tilde{J}\left(\mathbf{x}_{n}, \mathbf{x}_{d}\right) & \equiv \int_{B_{P} \cup B_{S}} W(\omega)|E(\omega)| d \omega \\
\text { subject to } \quad \hat{g}_{1}\left(\mathbf{x}_{n}, \mathbf{x}_{d}\right) & =0 \\
\hat{g}_{2}\left(\mathbf{x}_{n}, \mathbf{x}_{d}\right) & =0 \\
\hat{g}_{3}\left(\mathbf{x}_{d}\right) & =0 .
\end{aligned}
$$

However, problem $\mathbf{P}$ is still a nonsmooth nonconvex problem, where the nonsmooth function appears in the cost. Thus, standard optimization software packages, such as Matlab Optimization toolbox, in theory, cannot be applied directly. To overcome this difficulty, the nonsmooth absolute function $|E(\omega)| \forall \omega \in B_{P} \cup B_{S}$ is handled in the following manner. $\forall \omega \in B_{P} \cup B_{S}$ and $\varepsilon>0$, consider the following function:

$$
E_{\varepsilon}(\omega) \equiv\left\{\begin{array}{ll}
|E(\omega)| & |E(\omega)| \geq \frac{\varepsilon}{2} \\
\frac{(E(\omega))^{2}}{\varepsilon}+\frac{\varepsilon}{4} & |E(\omega)|<\frac{\varepsilon}{2}
\end{array} .\right.
$$

Clearly, the function $E_{\varepsilon}(\omega)$ possesses the following properties:

i) $\forall \omega \in B_{P} \cup B_{S}, \quad E_{\varepsilon}(\omega)$ is continuously differentiable with respect to $\left(\mathbf{x}_{n}, \mathbf{x}_{d}\right)$.

ii) $\forall\left(\mathbf{x}_{n}, \mathbf{x}_{d}\right)$ and $\forall \omega \in B_{P} \cup B_{S}, E_{\varepsilon}(\omega) \geq|E(\omega)|$.

iii) $\forall\left(\mathbf{x}_{n}, \mathbf{x}_{d}\right)$ and $\forall \omega \in B_{P} \cup B_{S},\left|E_{\varepsilon}(\omega)-\right| E(\omega)|| \leq(\varepsilon / 4)$.

iv) $\forall\left(\mathbf{x}_{n}, \mathbf{x}_{d}\right), \quad\left(\mathbf{x}_{n}^{*}, \mathbf{x}_{d}^{*}\right)$ minimizes $|E(\omega)|$ if and only if it minimizes $E_{\varepsilon}(\omega)$.

By virtue of these properties, $E_{\varepsilon}(\omega)$ is an ideal approximation of the nonsmooth function $|E(\omega)|$. By replacing $E_{\varepsilon}(\omega)$ for $|E(\omega)|$ in the cost function (25a), we obtain

$$
J_{\varepsilon}\left(\mathbf{x}_{n}, \mathbf{x}_{d}\right) \equiv \int_{B_{P} \cup B_{S}} W(\omega) E_{\varepsilon}(\omega) d \omega
$$

where the function $J_{\varepsilon}\left(\mathbf{x}_{n}, \mathbf{x}_{d}\right)$ is now continuously differentiable with respect to $\left(\mathbf{x}_{n}, \mathbf{x}_{d}\right), \quad \forall \varepsilon>0$. Hence, we can approximate the nonsmooth optimization problem $\mathbf{P}$ by a smooth optimization problem, where the cost function (27) is to be minimized subject to the equality constraints defined in (25b), (25c), and (25d). Let this optimization problem be referred to as problem $\mathbf{Q}_{\varepsilon}$ as follows.

Problem $\left(\mathbf{Q}_{\varepsilon}\right)$ :

$$
\begin{aligned}
\min _{\left(\mathbf{x}_{n}, \mathbf{x}_{d}\right)} \quad J_{\varepsilon}\left(\mathbf{x}_{n}, \mathbf{x}_{d}\right) & \equiv \int_{B_{P} \cup B_{S}} W(\omega) E_{\varepsilon}(\omega) d \omega \\
\text { subject to } \quad \hat{g}_{1}\left(\mathbf{x}_{n}, \mathbf{x}_{d}\right) & =0 \\
\hat{g}_{2}\left(\mathbf{x}_{n}, \mathbf{x}_{d}\right) & =0 \\
\hat{g}_{3}\left(\mathbf{x}_{d}\right) & =0 .
\end{aligned}
$$

$\forall \varepsilon>0$, let $\left(\mathbf{x}_{\varepsilon, n}^{*}, \mathbf{x}_{\varepsilon, d}^{*}\right)$ be an optimal solution to the approximate problem $\mathbf{Q}_{\varepsilon}$. Furthermore, let $\left(\mathbf{x}_{n}^{*}, \mathbf{x}_{d}^{*}\right)$ be an optimal solution to the original problem $\mathbf{P}$. Then, there are two questions to be answered. First, how much does $J_{\varepsilon}\left(\mathbf{x}_{\varepsilon, n}^{*}, \mathbf{x}_{\varepsilon, d}^{*}\right)$ differ from $\tilde{J}\left(\mathbf{x}_{n}^{*}, \mathbf{x}_{d}^{*}\right)$ ? Second, what is the relationship between $\left\{\left(\mathbf{x}_{\varepsilon, n}^{*}, \mathbf{x}_{\varepsilon, d}^{*}\right)\right\}$ and $\left\{\left(\mathbf{x}_{n}^{*}, \mathbf{x}_{d}^{*}\right)\right\}$ ? To address the first question, we have the following theorem.

Theorem 1: Let $\left(\mathbf{x}_{\varepsilon, n}^{*}, \mathbf{x}_{\varepsilon, d}^{*}\right)$ and $\left(\mathbf{x}_{n}^{*}, \mathbf{x}_{d}^{*}\right)$ be, respectively, optimal solutions to problems $\mathbf{Q}_{\varepsilon}$ and $\mathbf{P}$. Then $0 \leq J_{\varepsilon}\left(\mathbf{x}_{\varepsilon, n}^{*}, \mathbf{x}_{\varepsilon, d}^{*}\right)-$ $\tilde{J}\left(\mathbf{x}_{n}^{*}, \mathbf{x}_{d}^{*}\right) \leq \frac{\varepsilon}{4} \int_{B_{P} \cup B_{S}} W(\omega) d \omega$. 

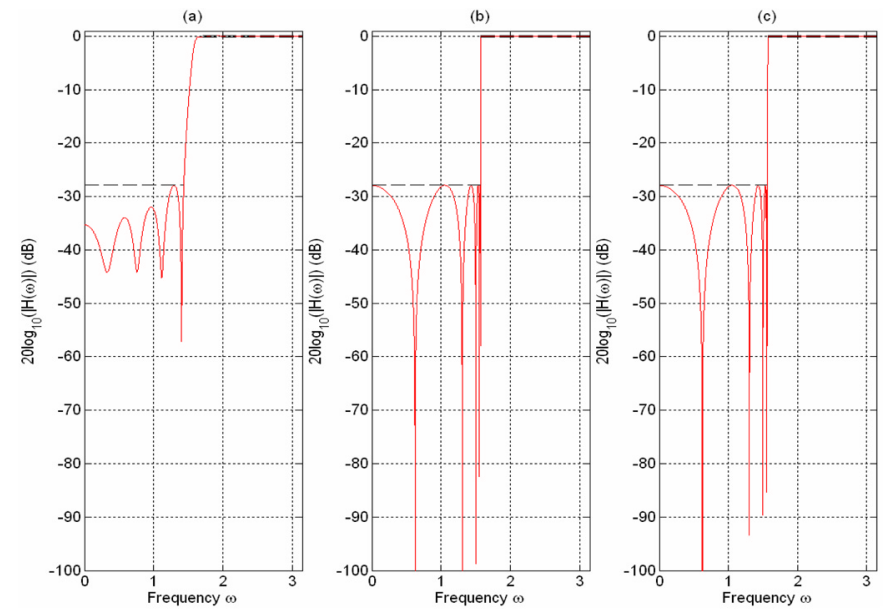

Fig. 2. Magnitude responses of various filters. (a) Filter designed via the iterative approach [9]. (b) Filter designed via an elliptic filter. (c) Filter designed via our proposed approach. All the passband and stopband ripple magnitudes are the same.

Proof: By virtue of property ii) of the function $E_{\varepsilon}(\omega)$, we have

$$
\begin{aligned}
J_{\varepsilon}\left(\mathbf{x}_{\varepsilon, n}^{*}, \mathbf{x}_{\varepsilon, d}^{*}\right) & \geq \tilde{J}\left(\mathbf{x}_{\varepsilon, n}^{*}, \mathbf{x}_{\varepsilon, d}^{*}\right) \geq \min _{\left(\mathbf{x}_{n}, \mathbf{x}_{d}\right)} \tilde{J}\left(\mathbf{x}_{n}, \mathbf{x}_{d}\right) \\
& =\tilde{J}\left(\mathbf{x}_{n}^{*}, \mathbf{x}_{d}^{*}\right) .
\end{aligned}
$$

Hence

$$
J_{\varepsilon}\left(\mathbf{x}_{\varepsilon, n}^{*}, \mathbf{x}_{\varepsilon, d}^{*}\right)-\tilde{J}\left(\mathbf{x}_{n}^{*}, \mathbf{x}_{d}^{*}\right) \geq 0 .
$$

Next, from property iii) of the function $E_{\varepsilon}(\omega)$, we have

$$
0 \leq J_{\varepsilon}\left(\mathbf{x}_{n}^{*}, \mathbf{x}_{d}^{*}\right)-\tilde{J}\left(\mathbf{x}_{n}^{*}, \mathbf{x}_{d}^{*}\right) \leq \frac{\varepsilon}{4} \int_{B_{P} \cup B_{S}} W(\omega) d \omega .
$$

However

$$
J_{\varepsilon}\left(\mathbf{x}_{\varepsilon, n}^{*}, \mathbf{x}_{\varepsilon, d}^{*}\right) \leq J_{\varepsilon}\left(\mathbf{x}_{n}^{*}, \mathbf{x}_{d}^{*}\right)
$$

so we have

$$
J_{\varepsilon}\left(\mathbf{x}_{\varepsilon, n}^{*}, \mathbf{x}_{\varepsilon, d}^{*}\right)-\tilde{J}\left(\mathbf{x}_{n}^{*}, \mathbf{x}_{d}^{*}\right) \leq \frac{\varepsilon}{4} \int_{B_{P} \cup B_{S}} W(\omega) d \omega .
$$

Hence, this completes the proof.

To address the second question, we have the following theorem:

Theorem 2: Let $\left\{\left(\mathbf{x}_{\varepsilon, n}^{*}, \mathbf{x}_{\varepsilon, d}^{*}\right)\right\}$ be a sequence of optimal solutions to the corresponding sequence of approximate problems $\left\{\mathbf{Q}_{\varepsilon}\right\}$. Then an accumulation point exists and it is an optimal solution to the original problem $\mathbf{P}$.

Proof: Since $J_{\varepsilon}\left(\mathbf{x}_{n}, \mathbf{x}_{d}\right)$ is continuous with respect to both $\left(\mathbf{x}_{n}, \mathbf{x}_{d}\right)$ and $\varepsilon,\left\{\left(\mathbf{x}_{\varepsilon, n}^{*}, \mathbf{x}_{\varepsilon, d}^{*}\right)\right\}$ is a convergent sequence and there exists an accumulation point $\left(\overline{\mathbf{x}}_{n}, \overline{\mathbf{x}}_{d}\right)$ and a subsequence of the sequence $\left\{\left(\mathbf{x}_{\varepsilon, n}^{*}, \mathbf{x}_{\varepsilon, d}^{*}\right)\right\}$, which is again denoted by the original sequence, such that $\left\|\left(\mathbf{x}_{\varepsilon, n}^{*}, \mathbf{x}_{\varepsilon, d}^{*}\right)-\left(\overline{\mathbf{x}}_{n}, \overline{\mathbf{x}}_{d}\right)\right\| \rightarrow 0$ as
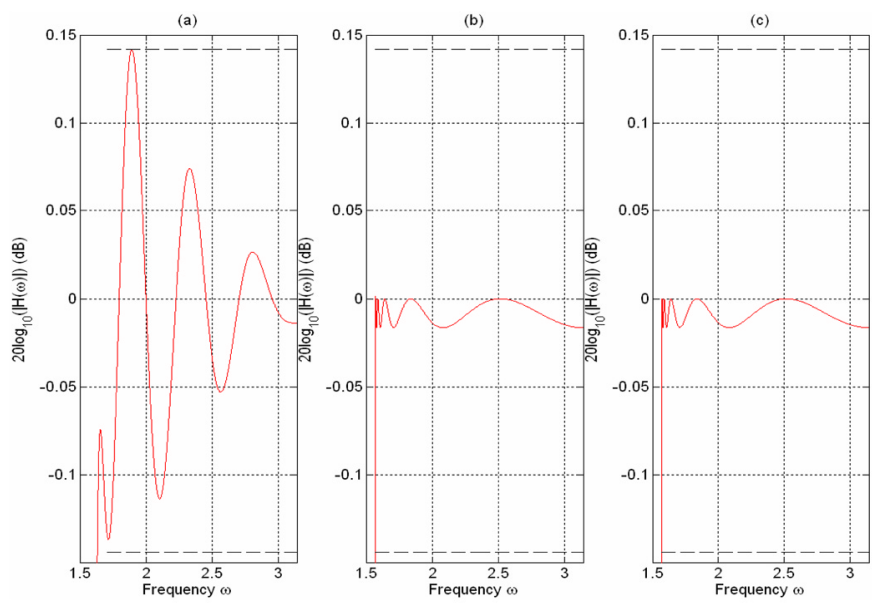

Fig. 3. Zoom of the magnitude responses in the passband. (a) Filter designed via the iterative approach [9]. (b) Filter designed via an elliptic filter. (c) Filter designed via our proposed approach. All the passband ripple magnitudes are the same.
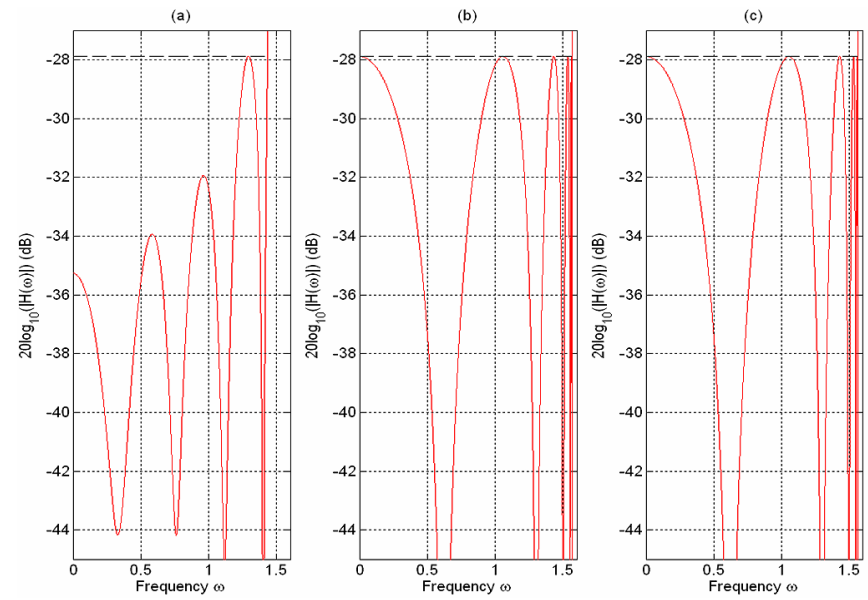

Fig. 4. Zoom of the magnitude responses in the stopband. (a) Filter designed via the iterative approach [9]. (b) Filter designed via an elliptic filter. (c) Filter designed via our proposed approach. All the stopband ripple magnitudes are the same.

$\varepsilon \rightarrow 0$, where $\|\cdot\|$ denotes the Euclidean norm. By Theorem 1, as $0 \leq J_{\varepsilon}\left(\mathbf{x}_{\varepsilon, n}^{*}, \mathbf{x}_{\varepsilon, d}^{*}\right)-\tilde{J}\left(\mathbf{x}_{n}^{*}, \mathbf{x}_{d}^{*}\right) \leq \frac{\varepsilon}{4} \int_{B_{P} \cup B_{S}} W(\omega) d \omega$, we have $J_{\varepsilon}\left(\mathbf{x}_{\varepsilon, n}^{*}, \mathbf{x}_{\varepsilon, d}^{*}\right) \rightarrow \tilde{J}\left(\mathbf{x}_{n}^{*}, \mathbf{x}_{d}^{*}\right)$ as $\varepsilon \rightarrow 0$. Hence, this completes the proof.

Based on these two theorems, problem $\tilde{\mathbf{P}}$ can be solved via solving a sequence of approximate problems $\left\{\mathbf{Q}_{\varepsilon}\right\}$ by an iterative technique stated in [24] with decreasing value of $\varepsilon$ and the algorithm is summarized as follows:

Algorithm 1:

Step 1) Initialize $\varepsilon_{1}>0$ and $k=1$.

Step 2) Solve problem $\mathbf{Q}_{\varepsilon_{k}}$ by hybrid global optimization method discussed in [25]. Denote the solution by $\left(\mathbf{x}_{\varepsilon_{k}, n}^{*}, \mathbf{x}_{\varepsilon_{k}, d}^{*}\right)$.

Step 3) Set $\varepsilon_{k+1}=\frac{\varepsilon_{k}}{L}$, where $L>1$ is a prespecified number.

Step 4) If $\left\|\left(\mathbf{x}_{\varepsilon_{k}, n}^{*}, \mathbf{x}_{\varepsilon_{k}, d}^{*}\right)-\left(\mathbf{x}_{\varepsilon_{k-1}, n}^{*}, \mathbf{x}_{\varepsilon_{k-1}, d}^{*}\right)\right\| \leq \beta$, where $\beta>0$ is a prescribed small number depending on the 

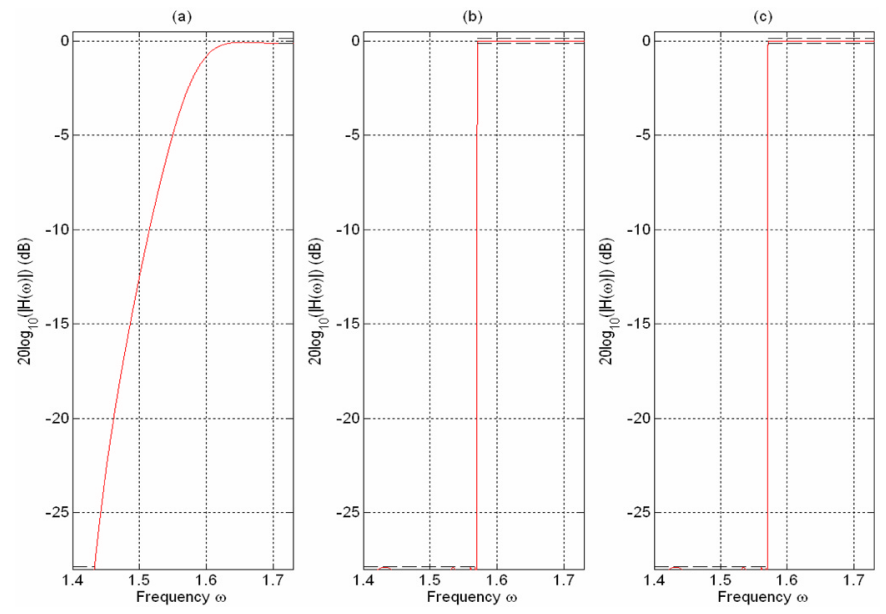

Fig. 5. Zoom of the magnitude responses in the transition band. (a) Filter designed via the iterative approach [9]. The transition bandwidth of the filter is 0.2756 . (b) Filter designed via an elliptic filter. The transition bandwidth of the filter is $1.382 \times 10^{-3}$. (c) Filter designed via our proposed approach. The transition bandwidth of the filter is $6.258 \times 10^{-4}$.

accuracy desired, then stop. Otherwise, set $k=k+1$ and go to Step 1).

In Algorithm 1, we can see that $\varepsilon_{k} \rightarrow 0$ as $k \rightarrow+\infty$ because $L>1$. Hence, according to Theorem 2 , we can see that the solution obtained $\left\{\left(\mathbf{x}_{\varepsilon, n}^{*}, \mathbf{x}_{\varepsilon, d}^{*}\right)\right\}$ converges to the global optimal solution of problem $\mathbf{P}$.

There are three parameters in the Algorithm 1, namely, $\varepsilon_{1}, L$ and $\beta$. $\varepsilon_{1}$ determines how close the approximate problem $\mathbf{Q}_{\varepsilon_{1}}$ and the original problem $\mathbf{P}$. The smaller the value of $\varepsilon_{1}$, the more close will be the problem $\mathbf{Q}_{\varepsilon_{1}}$ to problem $\mathbf{P}$, and hence the less number of iterations of Algorithm 1 is required. However, the cost function becomes less smooth. $L$ also determines the number of iterations required. Similarly, the larger the value of $L$, the less number of iterations is required, but the cost function becomes less smooth even for small values of $k$. Practically, we find that if $\varepsilon_{1} \approx 10^{-3}$ and $L \approx 10$, then the number of iterations required and the cost function will be, respectively, small and smooth enough for most optimization problems [24]. $\beta$ controls the acceptable precision of the obtained solution. The smaller the value of $\beta$, the more accurate of the solution is. However, the number of iterations required increases. Due to practical reasons, such as finite number of bits for representing filter coefficients, if $\beta \approx 10^{-6}$, then the obtained solution will be good for most applications [1]-[3].

It is worth noting that problem $\mathbf{Q}_{\varepsilon_{k}}$ is a nonconvex problem, so global optimal solution will not be guaranteed if it is solved via the existing gradient approach method [18]. In order to solve this difficulty, the hybrid global optimization method is applied [25] and is summarized as follows: The hybrid global optimization method consists of two basic components: local optimizers and feasible point finders. Given a feasible point, local optimizers will quickly produce a local optimal solution in the neighborhood of the feasible point. For the feasible point finders, first, choose an initial point $\left(\mathbf{x}_{\varepsilon_{k}, n}^{\circ}, \mathbf{x}_{\varepsilon_{k}, d}^{\circ}\right)$ from the feasible set of the problem $\mathbf{Q}_{\varepsilon_{k}}$ and start the local optimizer. Assume that the local optimizer has produced the local optimal solution of $J_{\varepsilon_{k}}\left(\mathbf{x}_{n}, \mathbf{x}_{d}\right)$ near $\left(\mathbf{x}_{\varepsilon_{k}, n}^{\circ}, \mathbf{x}_{\varepsilon_{k}, d}^{\circ}\right)$. Denote the local optimal solution and the corresponding local optimal value as $\left(\mathbf{x}_{\varepsilon_{k}, n}^{\bullet}, \mathbf{x}_{\varepsilon_{k}, d}^{\bullet}\right)$ and $J_{\varepsilon_{k}}\left(\mathbf{x}_{\varepsilon_{k}, n}^{\bullet}, \mathbf{x}_{\varepsilon_{k}, d}^{\bullet}\right)$. Then a new optimization problem with the same cost function but an additional constraint
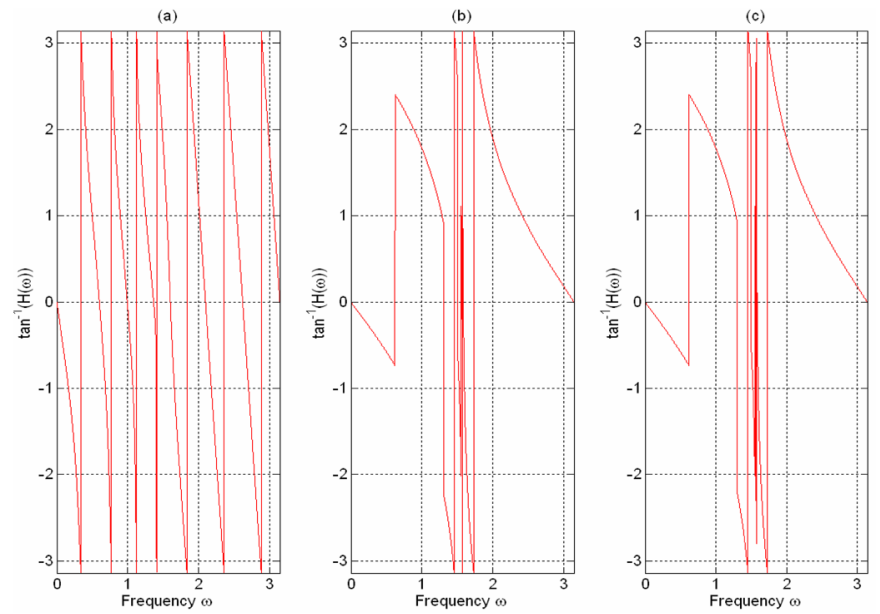

Fig. 6. Phase responses of various filters. (a) Filter designed via the iterative approach [9]. (b) Filter designed via an elliptic filter. (c) Filter designed via our proposed approach. The phase response of the filter designed using the iterative approach [9] is approximately linear, while those of via an elliptic filter and our design method are nonlinear.
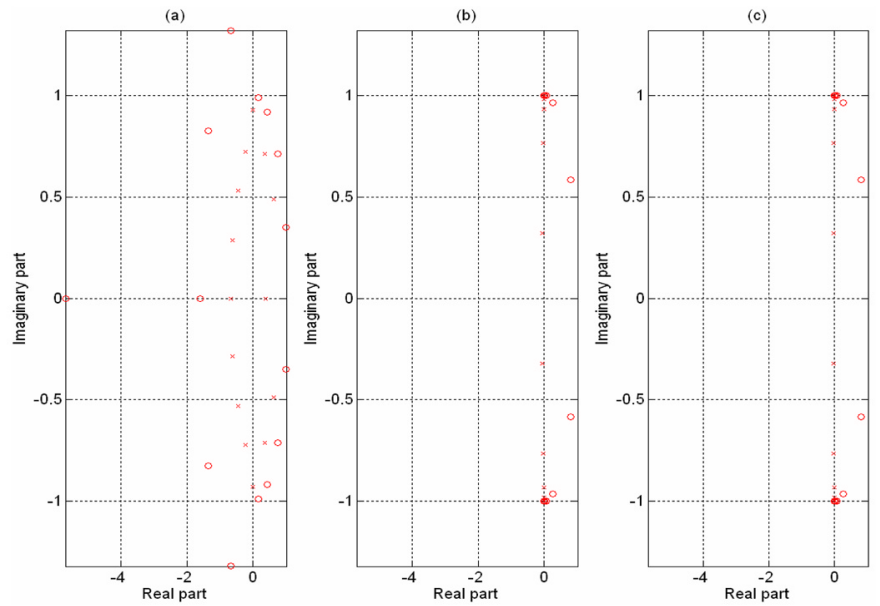

Fig. 7. Pole-zero plots of various filters. (a) Filter designed via the iterative approach [9]. (b) Filter designed via an elliptic filter. (c) Filter designed via our proposed approach. The poles and zeros of the filter designed using the iterative approach [9] are spread over a wide region in the complex plane, while those of the filters designed via an elliptic filter and our design method are located in a small region in the complex plane.

$J_{\varepsilon}\left(\mathbf{x}_{n}, \mathbf{x}_{d}\right)-J_{\varepsilon_{k}}\left(\mathbf{x}_{\varepsilon_{k}, n}^{\bullet}, \mathbf{x}_{\varepsilon_{k}, d}^{\bullet}\right)<0$ is added. Since the additional constraint is imposed in the optimization problem, the feasible set of this new optimization problem is smaller than that of the original optimization problem. Denote the new feasible set as $\bigcup_{j=0}^{l} S_{j}$. If the new optimization problem has no solution, then $\left(\mathbf{x}_{\varepsilon_{k}, n}^{\bullet}, \mathbf{x}_{\varepsilon_{k}, d}^{\bullet}\right)$ is taken as the global optimal solution of the original optimization problem. Otherwise, select $S_{j}$ from $\bigcup_{j=0}^{l} S_{j}$ and use the gradient and Newton method to find a feasible point in $S_{j}$ and restart the local optimal solution with this new initial feasible point. These procedures are repeated until a global optimal solution is obtained. In this hybrid global optimization algorithm, we can see that the feasible point finders serve two purposes: i) guarantee a solution that is better than the one obtained in the previous iteration; and most importantly and ii) if feasible point finders find no solution, then the global optimal 
TABLE I

Fitter Coefficients of Various Filters

\begin{tabular}{|c|c|c|c|c|c|}
\hline $\begin{array}{c}\text { Numerator } \\
\text { coefficients of filter } \\
\text { in [9] }\end{array}$ & $\begin{array}{c}\text { Denominator } \\
\text { coefficients of filter } \\
\text { in [9] }\end{array}$ & $\begin{array}{c}\text { Numerator } \\
\text { coefficients of an } \\
\text { elliptic filter }\end{array}$ & $\begin{array}{c}\text { Denominator } \\
\text { coefficients of an } \\
\text { elliptic filter }\end{array}$ & $\begin{array}{c}\text { Numerator } \\
\text { coefficients of our } \\
\text { designed filter }\end{array}$ & $\begin{array}{c}\text { Denominator } \\
\text { coefficients of our } \\
\text { designed filter }\end{array}$ \\
\hline 0.00216452 & 1 & 0.14216384559483 & 1 & 0.14209728097632 & 1 \\
\hline 0.01422263 & 0.85183505 & -0.33386656595210 & 0.12993638934053 & -0.33303728431125 & 0.13466225640668 \\
\hline 0.01070862 & 1.40277206 & 1.17827982115402 & 5.51295641219684 & 1.17628228645335 & 5.51353217578228 \\
\hline-0.00173896 & 1.15151797 & -2.02956611583674 & 0.67443232223880 & -2.02389980294615 & 0.69650844908505 \\
\hline-0.00056474 & 0.95948743 & 3.90213338343642 & 12.68806334048509 & 3.89299890676338 & 12.69055280095869 \\
\hline 0.01648599 & 0.81499579 & -5.11347674406074 & 1.44167986327036 & -5.09827278525309 & 1.48339221237488 \\
\hline 0.01450372 & 0.68685173 & 6.81014800154999 & 15.67113003662362 & 6.79226627722614 & 15.67539561982942 \\
\hline-0.01424925 & 0.52355787 & -6.83555438838710 & 1.62037059531367 & -6.81482053324876 & 1.66075436190002 \\
\hline-0.01101010 & 0.35584783 & 6.81014800154999 & 11.06560681872877 & 6.79226627722614 & 11.06922930471550 \\
\hline 0.04991283 & 0.23561367 & -5.11347674406074 & 1.00631571864580 & -5.09827278525310 & 1.02707195172405 \\
\hline 0.04637753 & 0.15595331 & 3.90213338343642 & 4.35630309971892 & 3.89299890676339 & 4.35783717002457 \\
\hline-0.19765241 & 0.08260605 & -2.02956611583674 & 0.32569583211244 & -2.02389980294615 & 0.33088092780985 \\
\hline 0.33139538 & 0.01749834 & 1.17827982115402 & 0.83772771965056 & 1.17628228645335 & 0.83799800124816 \\
\hline-0.25569216 & -0.01347975 & -0.33386656595210 & 0.04256677838515 & -0.33303728431125 & 0.04301731830653 \\
\hline 0.13691517 & -0.01109415 & 0.14216384559483 & 0.05100833029873 & 0.14209728097632 & 0.05101514127115 \\
\hline
\end{tabular}

solution will be found. Therefore, the hybrid global optimization method can always correctly find the global optimal solution. For the details, we recommend the readers to study [25].

Although the hybrid global optimization method [25] guarantees the global optimal solution, the rate of convergence of the algorithm depends on the initial choice of $\left(\mathbf{x}_{\varepsilon_{k}, n}^{\circ}, \mathbf{x}_{\varepsilon_{k}, d}^{\circ}\right)$. In order to have a fast rate of convergence, $\left(\mathbf{x}_{\varepsilon_{k}, n}^{\circ}, \mathbf{x}_{\varepsilon_{k}, d}^{\circ}\right)$ should be selected as close to the global optimal solution. For the rational IIR filter design problems, the solutions obtained using the elliptic filter design method may be a good choice of this initial guess because the solution obtained by the elliptic filter design method is a suboptimal solution.

\section{NUMERICAL EXPERIMENTS}

In this correspondence, a unit dc gain highpass halfband filter, that is $\tilde{H}(\omega)=\left\{\begin{array}{ll}1 & \omega \in B_{P} \\ 0 & \omega \in B_{s}\end{array}\right.$, where $B_{P}=[-\pi,-(\pi / 2)-\Delta] \cup$ $[(\pi / 2)+\Delta, \pi]$ and $B_{S}=[-(\pi / 2)+\Delta,(\pi / 2)-\Delta]$, in which $2 \Delta$ denotes the transition bandwidth of the filter, is designed for the illustration of the effectiveness of the proposed method. Halfband filters with unit dc gain are selected for illustration because they are found in many engineering applications, such as in wavelet applications. For other filters with different dc gains, such as lowpass filters, bandpass filters, band reject filters, notch filters, highpass filters with other passbands and stopbands, the design method can be applied directly.

To evaluate the effectiveness of the proposed method, our result is compared with the one obtained using the iterative approach [9] and that using an elliptic filter. These two design methods are chosen for comparisons because that using the iterative approach [9] would be of great value for the readers working in this field, while that using an elliptic filter because the design objectives are the same. For the iterative design approach, it was reported in [9] that the magnitude response of the filter in the passband and stopband is approximately bounded by, respectively, $0.1406 \mathrm{~dB}$ and $-27.8974 \mathrm{~dB}$, if the filter order is 14 . The corresponding magnitude response is shown in Fig. 2(a), the zoom in the passband, stopband and the transition band are shown in, respectively, Figs. 3(a), 4(a), and 5(a). It can be seen from the figure that the transition bandwidth is 0.2756 . To compare this result with that using an elliptic filter, we use the Matlab function "ellip" to implement the filter and set the filter order, as well as the passband and stopband specifications same as that reported in [9]. The corresponding magnitude response is shown in Fig. 2(b), while the zoom in the passband, stopband, and the transition band are shown in, respectively, Figs. 3(b), 4(b), and 5 (b). It can be seen from the figure that the transition bandwidth of the filter is $1.382 \times 10^{-3}$. For our design, we set both $W(\omega)=1$ and $\tilde{W}(\omega)=1, \forall \omega \in B_{P} \cup B_{S}$ for simplicity reasons. In fact, other positive weighting functions can be applied directly. For the parameters in the algorithm, we choose $\varepsilon_{1}=10^{-3} L=10, \beta=10^{-6}$, and $\left(\mathbf{x}_{\varepsilon_{1}, n}^{\circ}, \mathbf{x}_{\varepsilon_{1}, d}^{\circ}\right)$ as the elliptic filter coefficients as discussed in Section II. After running three iterations, the optimization algorithm terminates because the stopping criterion satisfies. The magnitude response of the filter is shown in Fig. 2(c), while the zoom in the passband, stopband, and the transition band are shown in, respectively, Figs. 3(c), 4(c), and 5(c). The phase responses and the pole-zero plots of these designed filters are shown in, respectively, Figs. 6 and 7, while the filter coefficients are listed in Table I. It can be checked that the transition bandwidth of our designed filter is $6.258 \times 10^{-4}$, which is $0.2271 \%$ of that using the iterative approach and $45.2822 \%$ of that using an elliptic filter. Our result performs much better than that using the iterative design approach [9] because this design approach requires a desired phase response and this information is necessary and cannot be removed from the design procedure. By a imposing an extra phase response on the design desired, the magnitude response will be the tradeoff. Our result also performs better than that using an elliptic filter because the one obtained using an elliptic filter is a local optimal solution, while our result is a global optimal solution.

It is worth noting that our proposed design method can be applied to a strong specification if a solution exists. Since there is a tradeoff between a filter length and a reduction on the passband and stopband ripple magnitudes, there does not exist any design that gives a filter with very short filter length but very large reduction on the passband and stopband ripple magnitudes. If there exists a stable filter such that it satisfies the specifications on the passband and stopband ripple magnitudes at a relatively short filter length, then a global optimal solution for the optimization problem exists. Since our proposed design method guarantees to obtain the global optimal solution, our proposed design method works properly under a strong specification if a solution exists. 
In order to test the rate of convergence of the algorithm, four different initial guesses of $\left(\mathbf{x}_{\varepsilon_{1}, n}^{\circ}, \mathbf{x}_{\varepsilon_{1}, d}^{\circ}\right)$ are used. These four initial guesses give the same global optimal solution. The design time for choosing $\left(\mathbf{x}_{\varepsilon_{1}, n}^{\circ}, \mathbf{x}_{\varepsilon_{1}, d}^{\circ}\right)$ as the elliptic filter coefficients is 2 seconds, that as the Chebyshev Type I filter coefficients is $10 \mathrm{~min}$, that as the Chebyshev Type II filter coefficients is $15 \mathrm{~min}$, and that as the one obtained using the iterative approach [9] is $1.5 \mathrm{~h}$, where all numerical experiments are running using a PC with Pentium 1.2-GHz CPU and 256-MB DDRAM. From these results, we can conclude that the required design time will be shorter if the initial guess is closer to the global optimal solution.

\section{CONCLUSION}

The main contribution of this correspondence is to formulate an optimum rational IIR filter design problem as a nonsmooth nonconvex optimization problem subject to continuous functional constraints. Our previous proposed constraint transcription method is applied to transform the continuous functional constraints to equality constraints. A hybrid global optimization method is applied to find the global optimal solution. According to our numerical experiments, small transition bandwidth filters are obtained.

\section{REFERENCES}

[1] S. S. Abeysekera, Y. Xue, and C. Charoensak, "Design of optimal and narrow-band Laguerre filters for sigma-delta demodulators," IEEE Trans. Circuits Syst. II, Analog Digit. Signal Process., vol. 50, no. 7, pp. 368-375, 2003.

[2] S. Bjærum, H. Torp, and K. Kristoffersen, "Clutter filter design for ultrasound color flow imaging," IEEE Trans. Ultrason., Ferroelectr., Freq. Control, vol. 49, no. 2, pp. 204-216, 2002.

[3] L. S. H. Ngia, "Recursive identification of acoustic echo systems using orthonormal basis functions," IEEE Trans. Speech Audio Process., vol. 11, no. 3, pp. 278-293, 2003.

[4] S.-C. Pei and J.-J. Shyu, "Design of 1-D and 2-D IIR eigenfilters," IEEE Trans. Signal Process., vol. 42, no. 4, pp. 962-966, Apr. 1994.

[5] — , "Eigenfilter design of 1-D and 2-D IIR digital all-pass filters," IEEE Trans. Signal Process., vol. 42, no. 4, pp. 966-968, Apr. 1994.

[6] F. Argenti and E. Del Re, "Design of IIR eigenfilters in the frequency domain," IEEE Trans. Signal Process., vol. 46, no. 6, pp. 1694-1698, Jun. 1998.

[7] X. Zhang and H. Iwakura, "Design of IIR digital allpass filters based on eigenvalues problem," IEEE Trans. Signal Process., vol. 47, no. 2, pp. 554-559, Feb. 1999.

[8] T. Matsunaga, M. Yoshida, and M. Ikehara, "Design of IIR digital filters in the complex domain by transforming the desired response," IEEE Trans. Signal Process., vol. 52, no. 7, pp. 1975-1982, Jul. 2004.

[9] W.-S. Lu, S.-C. Pei, and C.-C. Tseng, "A weighted least-squares method for the design of stable 1-D and 2-D IIR digital filters," IEEE Trans. Signal Process., vol. 46, no. 1, pp. 1-10, Jan. 1998.

[10] P. A. Regalia, "Comments on 'A weighted least-squares method for the design of stable 1-D and 2-D IIR digital filters'," IEEE Trans. Signal Process., vol. 47, no. 7, pp. 2063-2065, Jul. 1999.

[11] W.-S. Lu, S.-C. Pei, and C.-C. Tseng, "Reply to "Comments on 'A weighted least-squares method for the design of stable 1-D and 2-D IIR digital filters'," IEEE Trans. Signal Process., vol. 47, no. 7, p. 2066, Jul. 1999.
[12] W.-S. Lu, "Design of stable IIR digital filters with equiripple passbands and peak-constrained least-squares stopbands," IEEE Trans. Circuits Syst. II, Analog Digit. Signal Process., vol. 46, no. 11, pp. 1421-1426, 1999.

[13] C.-C. Tseng and S.-C. Pei, "Stable IIR notch filter design with optimal pole placement," IEEE Trans. Signal Process., vol. 49, no. 11, pp. 2673-2681, Nov. 2001.

[14] W.-S. Lu, "A unified approach for the design of 2-D digital filters via semi-definite programming," IEEE Trans. Circuits Syst. I, Fundam. Theory Appl., vol. 49, no. 6, pp. 814-826, 2002.

[15] C.-C. Tseng, "Design of IIR digital all-pass filters using least $p$ th phase error criterion," IEEE Trans. Circuits Syst. II, Analog Digit. Signal Process., vol. 50, no. 9, pp. 653-656, 2003.

[16] C.-C. Tseng, "Design of stable IIR digital filter based on least p-power error criterion," IEEE Trans. Circuits Syst. I, Reg. Papers, vol. 51, no. 9, pp. 1879-1888, 2004

[17] L. Li, L. Xie, W.-Y. Yan, and Y. C. Soh, "Design of low-order linear-phase IIR filters via orthogonal projection," IEEE Trans. Signal Process., vol. 47, no. 2, pp. 448-457, Feb. 1999.

[18] M. C. Lang, "Least-squares design of IIR filters with prescribed magnitude and phase responses and a pole radius constraint," IEEE Trans. Signal Process., vol. 48, no. 11, pp. 3109-3121, Nov. 2000.

[19] A. Tarczyñski, G. D. Cain, E. Hermanowicz, and M. Rojewski, "A WISE method for designing IIR filters," IEEE Trans. Signal Process., vol. 49, no. 7, pp. 1421-1432, Jul. 2001.

[20] X. Zhang, "Closed-form design of maximally flat IIR half-band filters," IEEE Trans. Circuits Syst. II, Analog Digit. Signal Process., vol. 49, no. 6, pp. 409-417, Jun. 2002.

[21] C. K. S. Pun, S. C. Chan, K. S. Yeung, and K. L. Ho, "On the design and implementation of FIR and IIR digital filters with variable frequency characteristics," IEEE Trans. Circuits Syst. II, Analog Digit. Signal Process., vol. 49, no. 11, pp. 689-703, Nov. 2002.

[22] B. Dumitrescu and R. Niemistö, "Multistage IIR filter design using convex stability domains defined by positive realness," IEEE Trans. Signal Process., vol. 52, no. 4, pp. 962-974, Apr. 2004.

[23] Y. Liu and K. L. Teo, "A bridging method for global optimization," $J$. Austral. Mathemat. Soc. Series B, vol. 41, pp. 41-57, 1999.

[24] K. L. Teo and C. J. Goh, "On constrained optimization problems with nonsmooth cost functionals," Appl. Math. Optim., vol. 18, pp. 181-190, 1988.

[25] P. Xu, "A hybrid global optimization method: The multi-dimensional case," J. Comput. Appl. Math., vol. 155, pp. 423-446, 2003. 